\title{
Modal Analysis of Vehicle-borne Sights Based on ANSYS Wu Shou-jun ${ }^{1}$, Ren Guo-quan ${ }^{1} \&$ Li Dong-wei ${ }^{1}$ \\ ${ }^{1}$ Department of Vehicle \& Electrical Engineering, Mechanical Engineering College, Shijiazhuang Hebei 050003, China
}

Keywords: vehicle-borne sights, ANSYS, finite element model, modal analysis

\begin{abstract}
According to static load standards and requirements of the vehicle sights mechanical structure, the paper makes the analysis of the structure characteristics of vehicle-borne sights, builds three-dimensional model of vehicle sights in UG and finite element model of the primary reflector in ANSYS. The theoretical modal analysis and experimental modal analysis of the primary reflector are carried out based on vibration testing requirements for the dynamic load, and the results are compared between the two methods. The result of theoretical modal analysis could meet the engineering accuracy requirements. The research provides more convincing evidence for the optimization design of primary reflector structure parameters, also provides an effective tool for the anti-vibration design of primary reflector.
\end{abstract}

\section{Introduction}

Armored fighting vehicles and tanks are important equipment on battlefield; its effectiveness consists of three factors: firepower, mobility and protective ability. Fire control system is the precise fire weapon system of armored fighting vehicles, mainly includes tracking guidance aiming mirror system, fire control computer system and gun control subsystem. As the part of tracking guidance sight system, sight is divided into upper reflector stabilized sight and lower reflector stabilized sight. In order to improve the battlefield survivability and fire fighting ability of the armored fighting vehicles and tanks, it requires that the armored vehicle is able to observe, aim and shoot perfectly in the complex battlefield, so the study of tracking guidance aiming mirror subsystem received widespread attention.

Paper [1] aimed at the stabilization problem of the Low-Light-Level image in bottom-reflecting fire control system, a novel real-time high-precision electronic image stabilization system based on a gyroscope and block matching algorithm was proposed. Its overall structure was designed; the transformation equations between the carrier movement and image movement were derived on the basis of perspective imaging model; the image motion model and the gyroscope signal calibration method were given also. Paper [2] aimed the complex structure on the observation equipment, so the staff cannot offer the technical guarantee for new equipment. Mathematical tools are used to express optical components imaging laws; it studied the fretting theory and builds the fretting model. With the computer simulation technology, combined with optical fretting theory and practical problems, it produced a set of simulation software and showed the influence of optical parts fretting. Paper [3] based on an optical collimator, the focal length of CCD optical system was measured and the system accuracy was analyzed. Results showed that the test method is accurate and reliable and detection precision is high, for high precision sights. The test provided a method for testing the sight-line alteration of high precision sighting telescopes.

The study above mainly focused on the theoretical analysis, the actual equipment experiments are of high cost. The paper mainly uses the UG NX 6.0 to build primary reflector model and ANSYS to mesh and make modal analysis, in order to understand the vibration characteristics of upper reflector stabilized sight; we make the simulation analysis, which provides a reference for the further research. 


\section{Introduction of vehicle-borne sight}

As shown in Fig.1, it is the structure diagram of armored vehicles upper reflector stabilized sight, position torque motor and resolver are mounted on the $\mathrm{Y}$ axle, pitch control liquid floated gyroscope is mounted on the $\mathrm{X}$ axle, and honeycomb primary reflector is mounted on $\mathrm{J}$ axle. Liquid floated gyroscope is a sensitive element, which can detect the angular alteration of $\mathrm{X}$ axle and $\mathrm{Y}$ axle, the angular signal can adjust and amplify the control system, and then drives torque motor on the $\mathrm{X}$ axle and $\mathrm{Y}$ axle to maintain stability, and rotates $\mathrm{J}$ axle through mechanical transmission, so as to achieve the purpose of stabilizing sight line [4].

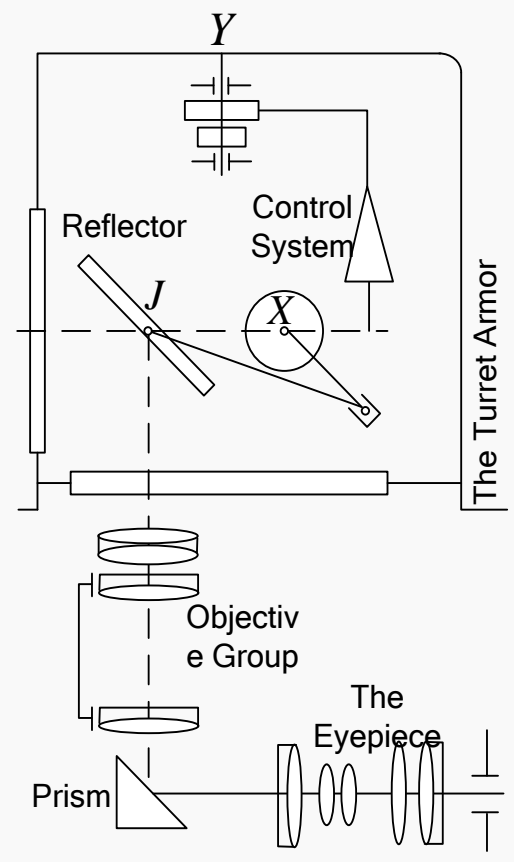

Fig.1: Simple sketch of upper reflector stabilized sight

\section{Build the finite element model}

\subsection{Build the geometric model}

As shown in Fig.2, we build three-dimensional model of primary reflector in UG NX6.0.
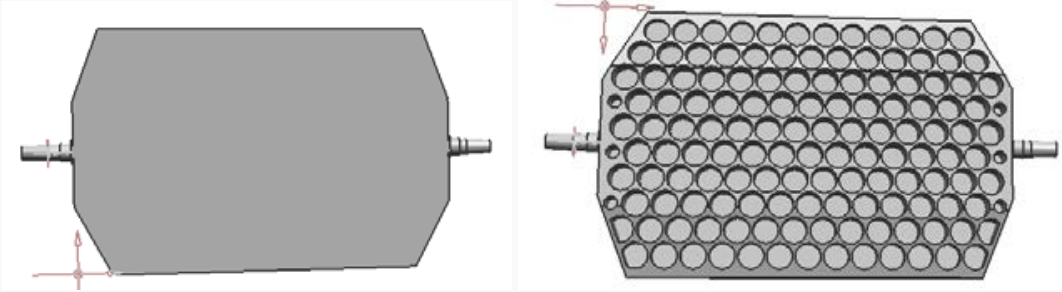

Fig.2: Model of primary reflector

\subsection{Mesh Division}

Mesh division is a key step in the process of finite element analysis, it includes three steps: unit properties defining, grid control parameters assigning, and grids generating. In order to improve the accuracy of the simulation analysis, it needs to define an accurate and appropriate unit property to each unit of aiming sight; property includes unit type, the real constant and material property. Material of reflector body is titanium alloy, its density is $4.45 \times 103 \mathrm{~kg} / \mathrm{m} 3$, elasticity modulus is $1.08 \times 105 \mathrm{MPa}$, Poisson's ratio is 0.34 , the reflector surface is plated with silver, the silver density is $10.5 \times 103 \mathrm{~kg} / \mathrm{m} 3$. By the method of general controlling the gridding density in ANSYS, it determines the unit size by defining the general unit number on boundary. Paper considers the curvature of line, empty proximity and unit order. The paper uses method of free mesh; there are no 
limit for cell shape, and no specific requirements on geometric model. This model select unit type Brick 20 node 186 in solid, unit number is 39491, node number is 74252, the finite element model is shown in Fig.3.

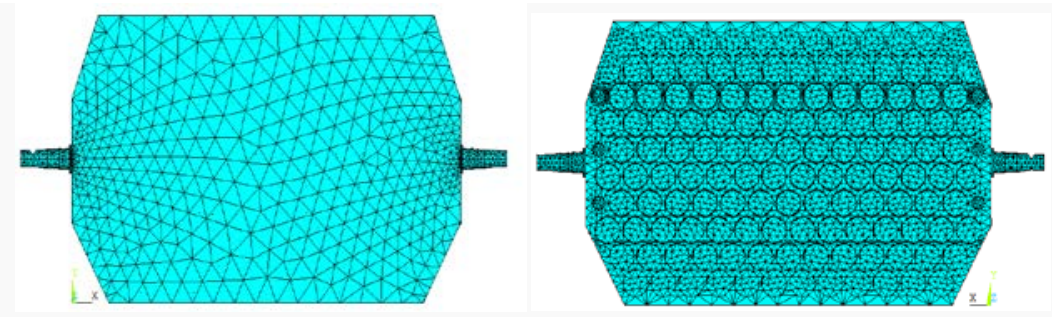

Fig.3: The finite element model

\section{Modal analysis}

As the natural vibration characteristics of mechanical structure, each order modal has a certain frequency and modal vibration mode. Modal analysis is a kind of method to study the dynamic characteristics of the structure, which can obtain the structure main modal characteristics of all orders in the susceptible frequency range, and can predict the actual vibration response in certain range of frequency. It assesses dynamic characteristics of the structure, and provides fundamental basis for the subsequent dynamic analysis and dynamic environment test [5].

\subsection{Theoretical basis [6-8]}

Structural dynamics differential equation is expressed as

$$
[\boldsymbol{M}]\{\ddot{\boldsymbol{q}}\}+[\boldsymbol{C}]\{\dot{\boldsymbol{q}}\}+[\boldsymbol{K}]\{\boldsymbol{q}\}=\{\boldsymbol{R}\}
$$

Where, $\{\boldsymbol{q}\}$ is the array composed of all the node displacements, $[\boldsymbol{M}] 、[\boldsymbol{C}] 、[\boldsymbol{K}]_{\text {are }}$ the mass matrix, damping matrix and stiffness matrix of the structure respectively, $\{\boldsymbol{R}\}$ is the node load array.

System inherent property has nothing to do with the external load, and the damping has little effect on natural frequency and vibration mode of the structure, so we can get the intrinsic characteristics of structure by calculating the free vibration equation. We get following equation from Eq. 1

$$
[\boldsymbol{M}]\{\ddot{\boldsymbol{q}}\}+[\boldsymbol{K}]\{\boldsymbol{q}\}=\mathbf{0}
$$

Its special solution is: $\{\boldsymbol{q}\}=\{\boldsymbol{\Psi}\} e^{j \omega t}$

Where $\omega$ is harmonic vibration frequency, $\{\boldsymbol{\Psi}\}$ is the node displacement amplitude vector. Take the special solution into Eq. 2, we get the free vibration differential equation written as

$$
\left([\boldsymbol{K}]-\omega^{2}[\boldsymbol{M}]\right)\{\boldsymbol{\Psi}\}=0
$$

We can obtain characteristic value $\omega_{1}{ }^{2} 、 \omega_{2}{ }^{2} 、 \cdots, \omega_{n}{ }^{2}$ and the corresponding feature vector $\left\{\boldsymbol{\Psi}_{1}\right\} 、\left\{\boldsymbol{\Psi}_{2}\right\}, \cdots,\left\{\boldsymbol{\Psi}_{n}\right\}$ from equation(3). The square root of characteristic value $\omega_{i}(i=1,2, \cdots, n)$ is the i-th order natural frequency of the structure; characteristic vector $\left\{\boldsymbol{\Psi}_{i}\right\}(i=1,2, \cdots, n)$ is the i-th order modal vibration mode of the structure.

\subsection{Theoretical modal analysis}

Use the finite element model to analyze natural frequency and modal vibration of the model. Modal analysis consists of three elements: natural frequency, mode shape and damping. Inherent frequency is the main vibration under a certain frequency, resonance occurs when the external excitation frequency is close to the certain natural frequency. Vibration mode reflects the vibration shape of the object in a certain natural frequency. Natural frequency and mode shape are two basic 
parameters to report the dynamic performances. Low-order vibration tends to concentrate the main energy of all the order. Therefore, the first six order natural frequencies and vibration mode values are meeting the requirements for general structure [9-10]. As shown in Table 1, build a finite element model of primary reflector in ANSYS and obtain first six order natural frequencies, the first six order modal vibration modes are shown in Fig.4.

Table 1: First six order natural frequency of primary reflector

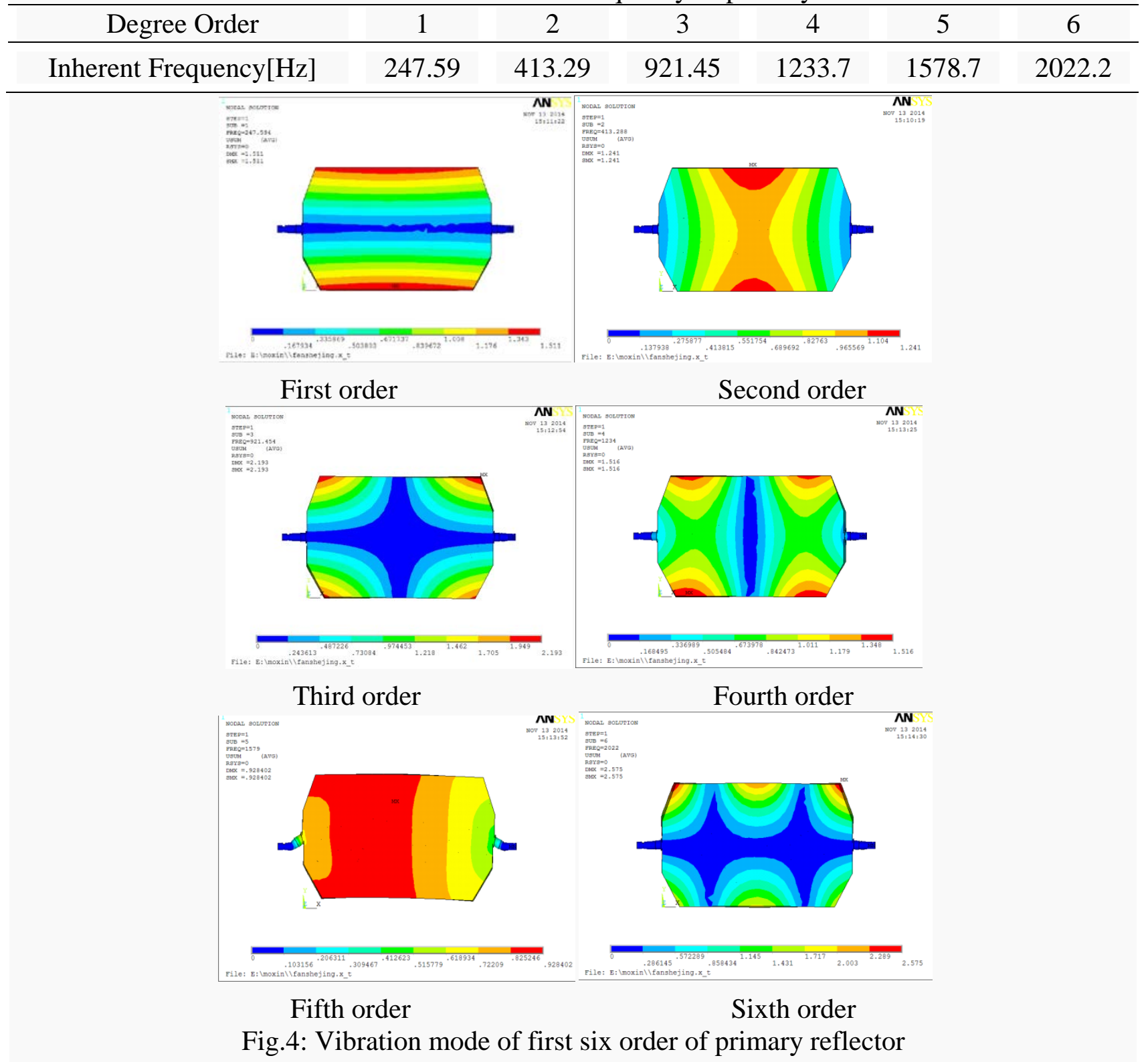

From Fig. 4 we can see that the first order natural frequency of the reflector is $247.59 \mathrm{~Hz}$, the second order natural frequency is $413.29 \mathrm{~Hz}$, the third order natural frequency is $921.45 \mathrm{~Hz}$, fourth order natural frequency is more than $1000 \mathrm{~Hz}$, it is usually not excited, so the first three order modal contribute to the response results much more than others. The vibration mode diagrams show that the deformation in bearing of each order modal is little. However, the larger deformation is produced on the middle edge of reflector, so the up and down edge of reflector are the key parts of strengthen.

\subsection{Experimental modal analysis}

Experimental modal analysis can not only reflect the dynamic characteristics of the sight structure, also can validate the theoretical analysis results. When analyze modal of a system, we commonly use the subspace iteration method and the Block Lanczos method, the two methods are 
suitable for the most modal analysis, yet asymmetric matrix and damping method are used in some special occasions [11-12]. Comparing the experimental modal analysis results to the theoretical frequency, we can verify the feasibility of finite element analysis, only the first few order natural frequencies and vibration modes are used for comparative analysis. Take sight as object, the prototype is fixed on the vibration table by the anchor bolts, to simulate the working conditions of bottom support structure. Test procedures prescribed by the GB/T 2423.10, in order to make the modal test results can truly reflect the actual characteristics of sights; we take all kinds of factors into consideration, such as the installation of sensors, fixed form of sights. Theoretical natural frequencies and experimental modal analysis results are shown in Table 2; the average error is $5.13 \%$.

Table 2 Modal analysis results

\begin{tabular}{ccccccc}
\hline Degree Order & 1 & 2 & 3 & 4 & 5 & 6 \\
\hline $\begin{array}{c}\text { Theoretical } \\
\text { Frequency[Hz] }\end{array}$ & 247.59 & 413.29 & 921.45 & 1233.7 & 1578.7 & 2022.2 \\
\hline $\begin{array}{c}\text { Experimental } \\
\text { Frequency[Hz] }\end{array}$ & 229.38 & 397.01 & 895.25 & 1168.0 & 1498.4 & 1926.6 \\
\hline Error [\%] & 7.9 & 4.1 & 2.9 & 5.6 & 5.3 & 2.9 \\
\hline
\end{tabular}

We can see from table 3 that there are certain errors between the theoretical results and experimental results, it is mainly because that simplified model of sight. Connection parts are different with the actual situation. But the results show that the maximum error of finite element analysis and modal test is no more than $10 \%$, the average error is only $5 \%$. Thus, the precision of the finite element model can meet the engineering requirement. The finite element model can reflect the actual prototype characteristics better, and be able to provide a certain reference for optimization of sight structure parameters.

\section{Conclusions}

Through the research of vehicle sights in work and transportation environment, we build the finite element model of reflector. The modal analysis is carried out on the primary reflector, we obtain each order natural frequency and vibration mode diagram of reflector, and compared with experimental modal analysis, modal analysis results show that the theoretical modal analysis of high accuracy, which can meet the engineering requirements. This paper makes a beneficial exploration for the optimization design of vehicle sights.

\section{References}

[1] Zong Yan-tao, Jiang Xiao-yu, Wang Xi, Ji Hong-xia, Zhao kai, Research on electronic image stabilization technique for vehicle-borne low-light-level telescope. Acta Armaentrii. 33(9),pp.1041-1046,2012.

[2] Li qiang, Zhang Xu-fan, Yan Xing-peng, Cheng Gao-feng, Simulation of fault of tank's sighting system due to fretting of optical parts, Science \& Technology Herald. 31(23),pp.22-25,2013.

[3] Lv Pu, Han Guo-hua, Zhang Ai-li, Jiang Xian-dong, Study on measurement of sight-line alteration of high precision sighting telescopes, Laser Technology. 37(3),pp.404-408, 2013.

[4] Chen Hai-hong, Wang Xing-qiao, Technical Comparison between Upper Reflector Stabilized Sight and Lower Reflector Stabilized Sight, Acta Armaentrii. 3,pp.49-52,1993.

[5] Chai Guo-dong, Dynamics analysis and thermal stress analysis of an airborne electronic equipment, Chengdu: university of electronic science and technology, 2008.

[6] Li-long, Modal and Stress Analysis of Certain Antenna Pedestal, Electro-Mechanical Engineering. 25(2),pp.62-64,2009.

[7] Liu Ren-fei, Zhang hua-jun, Static load and modal analysis of electronic cabinet based on finite element method, Electro-Mechanical Engineering. 
30(1),pp.11-13,2014.

[8] Wei Qiang, Li Yu, Dynamic structure optimization method based on FEM analysis, Electro-Mechanical Engineering. 26(2),pp.36-38,2010.

[9] Yu Yang, Yang Wen-fang, Dynamic simulation analysis for a ship-borne console structure, Electro-Mechanical Engineering. 27(6),pp.58-61,2011.

[10] Yang yu-jun, Vibration analysis of the aerospace rugged computer using ANSYS dynamic simulation technique, Electro-Mechanical Engineering. 19(5),pp.42-47,2003.

[11] Wang xian-zhou, Wang chang-wu, Simulation analysis of an airborne radar cabinet based on MD. Nastran, Electro-Mechanical Engineering. 27(1), pp.12-14,2011.

[12] Fang Wei-ji, Wang ke-jun, Zhang Ming, Analysis of vibration modes and frequency response of airborne electronic device, Electro-Mechanical Engineering. 26(5),pp.49-53,2010. 